\title{
A study on the operation of rehabilitation interfaces in active rehabilitation exercises for upper limb hemiplegic patients: Interfaces for lateral and bilateral exercises
}

\author{
Su-Hong Eom and Eung-Hyuk Lee* \\ Department of Electronic Engineering, Korea Polytechnic University, Siheung, Gyeonggi-Do, Korea
}

\begin{abstract}
.
BACKGROUND: For implementing autonomous rehabilitation exercises for upper limb hemiplegic patients, interfaces and a rehabilitation scenario that allow lateral and bilateral motions in a rehabilitation exercise robot are proposed.

OBJECTIVE: The proposed method measures the motion information generated from the unaffected part and projects it to an affected part in which the affected part expresses motions of the unaffected part.

METHODS: Both the accelerometer and gyro data were merged for estimating the motion information of the unaffected part. Also, HDR and complementary filters were applied to improve measurement errors in a data merging process.

RESULTS: For verifying the proposed method, a device, which is similar to a human body joint, was fabricated. Then, the angular values estimated by using an inertial sensor and the encoder values from the device were compared. In addition, a camera analysis was used to verify the proposed rehabilitation scenario by applying the rehabilitation interface proposed in this study to an exo-skeleton robot arm.

CONCLUSION: It is possible to apply the method proposed in this study to the control variables in different upper limb rehabilitation exercise robots. Thus, it is expected that patient centered active lateral/bilateral rehabilitation exercises can be performed through this interface method.
\end{abstract}

Keywords: Lateral and bilateral upper limb movement, upper limb rehabilitation, active rehabilitation, rehabilitation interface

\section{Introduction}

Functional abnormalities in the human body are represented by different types. It becomes a factor of inhibiting activities of daily living (ADL) and degrades the lives of human beings [1,2]. These abnormalities are ascertained as disabilities and specific surgeries and rehabilitation exercises are implemented to recover body functions. The rehabilitation exercise has traditionally been performed by rehabilitation therapists, occupational therapists, and others for activating nerves and muscles in a disordered part through systematic and consistent repetitive exercises [3-5,9]. However, it is difficult to exactly estimate the degree of rehabilitation in patients due to the fact that the rehabilitation exercise is performed by

\footnotetext{
${ }^{*}$ Corresponding author: Eung-Hyuk Lee, Department of Electronic Engineering Korea Polytechnic University, \#2121, Jeongwang-Dong, Siheung-City, Gyeonggi-Do, Korea. E-mail: ehlee@kpu.ac.kr.
}

0928-7329/16/\$35.00 (C) 2016 - IOS Press and the authors. All rights reserved This article is published online with Open Access and distributed under the terms of the Creative Commons Attribution NonCommercial License. 
simple repetitive actions and becomes passive exercises after a given period in addition to lacks of rehabilitation therapists. In order to solve these problems several robot based rehabilitation devices and products are investigated and developed and some cases are reported as useful rehabilitation results using these devices and products [5-9]. Also, studies on rehabilitation therapies using robots have been conducted in rehabilitation hospitals and therapies as different ways based on clinical effects [5-10].

As rehabilitation exercises using a rehabilitation robot represent different ways according to subjects and rehabilitation ranges, it is necessary to clearly define the subjects in rehabilitation exercises. In this study, the subjects are upper limb hemiplegic patients.

The hemiplegic symptom represents rigidities or degradations of muscle power in a part of the human body and is mainly caused by the damages of muscles and nerves because of accidents $[7,11]$. The upper limb hemiplegic patient groups are largely classified into upper and lower limb hemiplegia. As the upper limb has no close interaction between left and right parts compared to that of the lower limb, it may cause a termination of exercise capabilities in future if proper rehabilitation exercises for the affected part is not implemented [12]. Therefore, proper rehabilitation exercises are essential for these patients.

The rehabilitation exercise programs for upper limb hemiplegic patients are FES (Functional Electrical Stimulation), therapeutic exercises, Bobath therapies, and son on [10,13-15]. In these rehabilitation methods, the exercise programs performed by therapists are to be performed for a long period of time [16]. In reality, however, it is difficult to perform these close exercises due to lacks of rehabilitation therapists.

The practical problems that represent difficulties in close exercises can be solved using rehabilitation robots and this method has partly been used. However, the rehabilitation exercise robot and research operated in these days have been performed based on implementing multi-degree of freedom considering the intelligent and various motion ranges related to the safety of users compared to the conventional continuous passive movement (CPM). Also, its implementation can be performed as both the affected part exercise and the bilateral exercise $[3,5,7,17,18]$. However, it is difficult to implement autonomous exercises because these robots are considered as a type that improves the original function of conventional CPM devices. It is due to the fact that the rehabilitation exercise is implemented by a programmed rehabilitation sequence by attaching a rehabilitation robot (device) to an affected part or both parts or is performed by contents (games) in some intelligent rehabilitation robots [3,5-7,10]. Although the rehabilitation robot based on contents represents more interests in rehabilitation exercises than that of the rehabilitation exercise program based robot, it is difficult to reflect the continuous and active will of patients to the system because the contents based method becomes a passive exercise because of becoming familiar with such contents in a long term period of exercises.

Thus, in this study a rehabilitation exercise scenario and exercise interfaces that can maintain sustainable rehabilitation exercises while helps of rehabilitation therapists are to be minimized are proposed.

The proposed method is a rehabilitation interface method that uses the unaffected (healthy) arm of a hemiplegic patient. This method represents an expression of the estimated unaffected joint motion information of a upper limb hemiplegic patient to the joint of a rehabilitation robot attached to the affected part. It allows lateral exercises because it wears a rehabilitation exercise robot on the affected part and is possible to implement bilateral exercises because the affected part expresses motions of the unaffected part.

The bilateral rehabilitation exercise has been used as a rehabilitation exercise method that improves the bilateral cooperation of both hands in stroke patients [4,12]. The efficacy of rehabilitation exercises has been verified through different studies in improving both the range of motion and the exercise performance in the affected part $[3,4,11,12,19]$. However, the current robot based bilateral rehabilitation 
exercise dose not classify affected or unaffected parts and can be performed by wearing devices or robots on both arms. Although this method increases the effect of rehabilitation through the cooperation between unaffected and affected parts, it is not enough to induce interests or motivations of rehabilitation patients. For solving this issue a method in which the affected part represents motions of the unaffected part differing from the conventional rehabilitation method is proposed in this study. As this method shows visual effects of rehabilitation patients, it can induce interests and motivations during its rehabilitation process. It is verified that the visual effects improve rehabilitation performance in a mirror intermediate therapy for hemiplegic patients [20-22].

Therefore, in this study a rehabilitation interface method that maximizes the advantages of precedent rehabilitation studies is proposed to perform active rehabilitation exercises for upper limb hemiplegic patients.

For implementing the proposed interface method it is necessary to plan a rehabilitation scenario and to deduce requirements in rehabilitation motions based on the scenario. Then, a motion information module is designed based on it. In addition, a method of estimating the motion information of the unaffected part and a method of inducing control variables for operating a rehabilitation robot are to be proposed.

\section{Operation scenario of the proposed active rehabilitation exercise interface}

This study proposes a rehabilitation scenario of the lateral and bilateral exercise for autonomous rehabilitation exercise programs. The proposed scenario was established based on the consultation of the physical and occupational therapists of the national rehabilitation center (NRC) in Korea.

The rehabilitation exercise program consists of three steps and the forward step is determined by a rehabilitation therapist through considering the condition and fatigue level of patients. In Steps 2 and 3, the number of trials is controlled by the rehabilitation therapist based on the composition of motions.

The therapist analyzes the performance time and the muscle activity measured from the affected side in each step and determines the forward step according to exercise and improvement levels. Patients complete the scenario through these three steps. The duration for each step is limited by about $30 \mathrm{~min}-$ utes.

The rehabilitation process based on the induced rehabilitation scenario is presented in Fig. 1.

\section{Considerations and requirements in the estimation of body motions for rehabilitation exercises}

The interface method for the active rehabilitation exercise proposed in this study expresses the motion information of the unaffected part to the affected part differing from the conventional robot based method. Figure 2 shows the interface control block diagram of the upper limb rehabilitation robot proposed in this study.

In order to perform the method as shown in Fig. 2, it is necessary to precedently estimate the motion information of the brachium and forearm of the unaffected part using a motion recognition module. The estimated motion information will be used as the joint angle control variables of rehabilitation robots in future.

As represented in Fig. 3, the upper limb rehabilitation motions are divided into rising/falling arms to front, rising/falling arms to side, and outstretching and rotating arms including the motions of tucking elbows and rotating wrists [3-7,10,12]. In this study, however, the wrist rotation is not considered. 

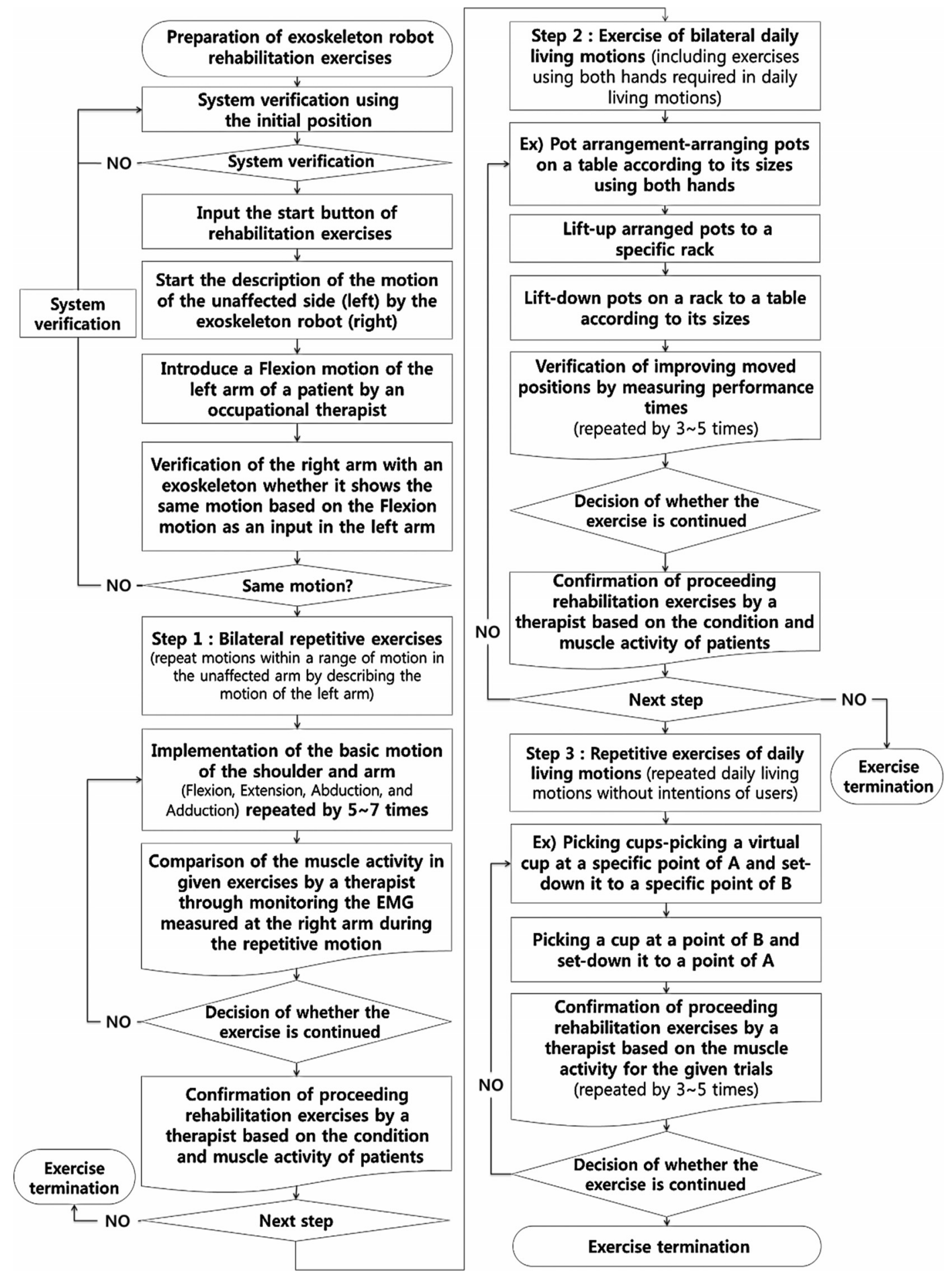

Fig. 1. The rehabilitation process based on the induced rehabilitation scenario. 
Table 1

Rehabilitation exercise step

\begin{tabular}{|c|c|c|}
\hline Step & Exercise & Description \\
\hline Step 1 & $\begin{array}{l}\text { Exercises of bilateral repeti- } \\
\text { tive motions } \\
\text { (Repetition } 5 \sim 7 \text { times) }\end{array}$ & $\begin{array}{l}\text { - Exercises of inducing the joint motion of the right arm using the healthy left } \\
\text { arm } \\
\text { - Performing joint motions for different directions compared to the existing } \\
\text { method by clasping hands }\end{array}$ \\
\hline Step 2 & $\begin{array}{l}\text { Exercises of bilateral daily } \\
\text { living motions } \\
\text { (Repetition } 3 \sim 5 \text { times) }\end{array}$ & $\begin{array}{l}\text { - Including exercises of the same motion and purpose using both hands } \\
\text { - Composing exercises based on a proper environment provided by a therapist } \\
\text { and a rehabilitation purpose of patients } \\
\text { - Composing exercises of bilateral motions for different purposes like arranging } \\
\text { pots, pulling draws, lifting pots, and etc. including daily living motions }\end{array}$ \\
\hline Step 3 & $\begin{array}{l}\text { Exercises of daily living mo- } \\
\text { tions } \\
\text { (Repetition } 3 \sim 5 \text { times) }\end{array}$ & $\begin{array}{l}\text { - Exercises of daily living motions using a single arm through repeating motions } \\
\text { included in exoskeletons } \\
\text { - Repetitive exercises of the motions recommended by a therapist using a robot, } \\
\text { such as answering phone calls, getting down objects using a single hand, lifting } \\
\text { objects using a single hand, and etc. }\end{array}$ \\
\hline
\end{tabular}

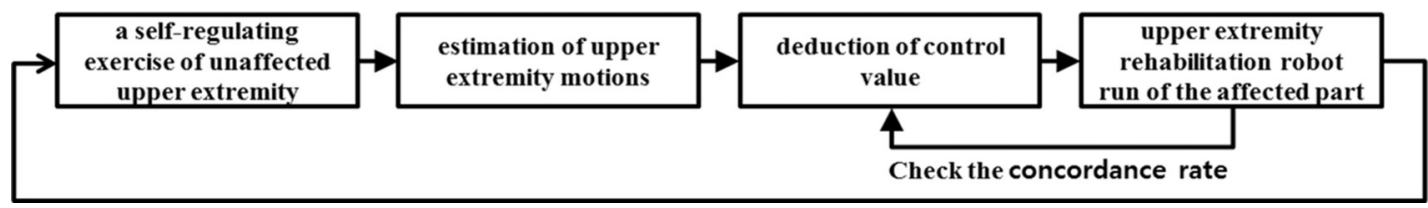

Fig. 2. Robot control method of upper extremity rehabilitation.
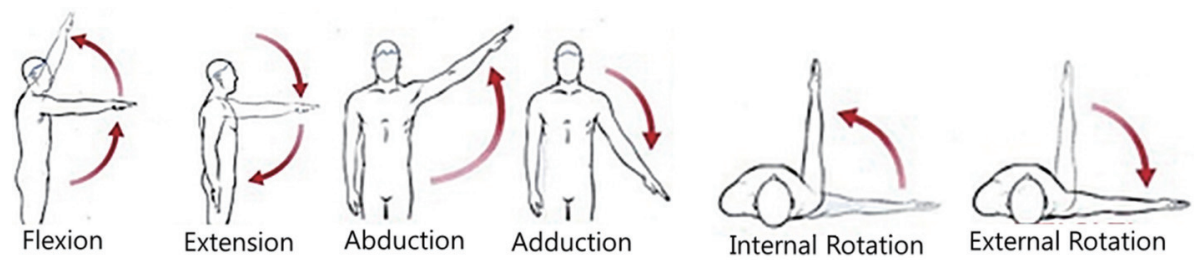

Fig. 3. Movement range of upper extremity.

For estimating these motions the elements of Roll, Pitch, and Yaw in three axes are to be considered and sensor modules are also designed through considering the place in which rehabilitation exercises are performed in a room. Thus, the motion estimation module proposed in this study consists of accelerometers and gyroscope sensors. In the case of commercial IMU modules, it merges terrestrial magnetism sensors and GPS information. However, in this study these are not included in the module because the rehabilitation exercises are performed in a room. The accuracy of the angle estimation required in this study is defined within a range $1^{\circ}$ and the angle estimation algorithm is defined as an algorithm with small arithmetics compared to the estimated accuracy based on the same reason of determining the estimation accuracy.

In the estimation of positions, the Flexion/Extension and Abduction/Adduction motions can be detected using accelerometers and gyroscope sensors alone or in its combination. However, the Internal/ External Rotation motions show limitations in estimating it using an accelerometer based on the rotation of the Yaw axis. Thus, the motions are to be detected using the gyroscope sensor only. However, it requires a preprocessing process in order to minimize bias values, which are presented by a system 


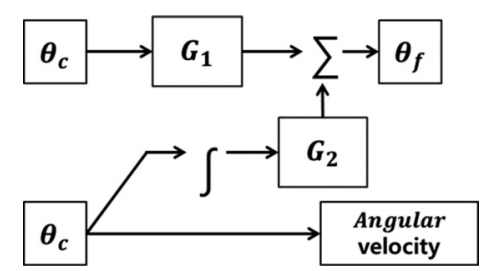

Fig. 4. The concept of complementary filter.

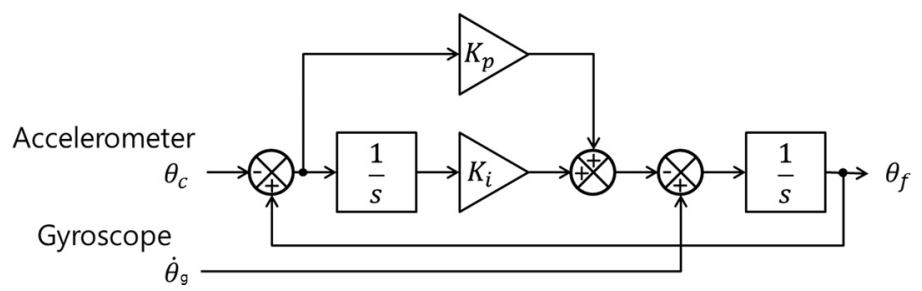

Fig. 5. 2nd complementary filter which applied proportional integtator.

process, a module fabrication process, and temperature, before estimating angles [23].

For implementing the rehabilitation motion that requires elbow motions it is necessary to estimate the elbow joint angles. The elbow joint is a lower joint of the shoulder joints and is a connection link between the brachium and the forearm. The estimation of the elbow joint requires a relative estimation method for the shoulder joint because the center of rotation in the joint is varied according to changes in the shoulder as the estimation is carried out using an inertial sensor.

\section{Method}

\subsection{Design of an inertial measurement device based motion recognition module}

The inertial measurement device proposed in this study consists of a three-axis (3DoF) gyroscope and a three-axis (3DoF) accelerometer. The accelerometer produces instantaneous acceleration and deceleration or acceleration caused by gravity. However, it is not possible to detect instantaneous changes in such acceleration and deceleration as a time of obtaining data in sensors. It can be implemented as an analysis of high frequency noise [24]. A gyroscope obtains angle values by integrating angular velocity. This calculation method may generate cumulative errors because the drift in a gyroscope is integrated during the calculation of angle values and the errors are considered as an induction of low frequency noise $[23,25,26]$. Thus, in this section the errors are compensated using a complementary filter that complements mutual disadvantages in acceleration and a gyroscope. Figure 4 represents a primary complementary filter $[27,28]$.

The complementary filter shown in Fig. 4 is not recognized by the previously mentioned low and high frequency pass filters and adds the angle value obtained by acceleration with a weigh value to the angular velocity of a gyroscope. It is a feedback structure according to time. In a mathematical approach, it is a combination of low and high frequency pass filters. The mathematical approach is to be considered in the secondary complementary filter.

In this study a secondary complementary filter with a differentiation-integrator is designed to compensate the basic complementary filter. It is due to the fact that the damping ratio in cutoff frequency is increased according to increases in the order of frequency pass filters.

The secondary complementary filter applies the differentiation-integrator as an input and is designed to correct errors in the angular velocity of a gyroscope. Figure 5 shows the block diagram of the complementary filter using the differentiation-integrator.

As shown in Fig. 5, it is a type that estimates the final angle by integrating the compensated angular velocity in which the output of the differentiation-integrator in the angular velocity measured using a gyroscope is excluded. Equation (1) shows the secondary complementary filter using the differentiationintegrator. 


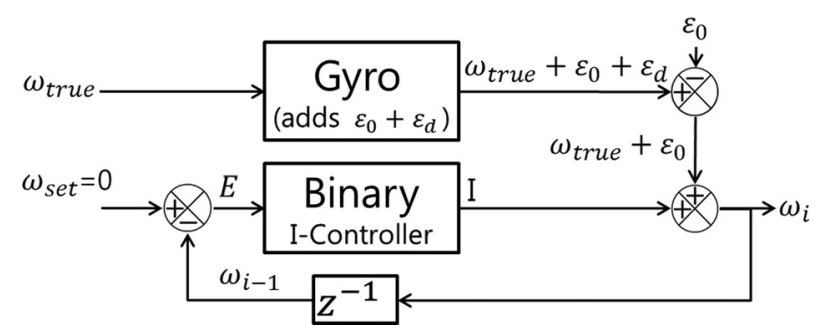

Fig. 6. The concept of HDR filter.

It shows that it is determined by low and high frequency pass filters based on Eq. (2) and is the same idea as the complementary filter.

$$
\begin{aligned}
& \theta_{f}=\frac{1}{S}\left[\theta_{g}-\left(K_{p}+\frac{1}{S} K_{i}\right)\left(\theta_{f}-\theta_{c}\right)\right] \\
& \theta_{f}=\frac{S^{2}}{S^{2}+K_{p} S+K_{i}}\left(\frac{1}{S} \theta_{g}\right)+\frac{K_{p} S+K_{i}}{S^{2}+K_{p} S+K_{i}} \theta_{c}
\end{aligned}
$$

\subsection{The method of drift compensation of gyroscope sensor}

Although upper limb positions in different upper limb motions can be detected using the previously mentioned complementary filter, it is difficult to ensure the accuracy in internal and external rotation motions based on acceleration sensors. Thus, the portion of the gyro sensor data is to be increased in such internal and external rotation motions. However, the gyro sensor generates integration errors due to various reasons [23]. For improving these errors, a heuristic drift reduction (HDR) filter is used to remove bias values for each sampling time in order to reduce the errors during the merging process of the complementary filter based on the improvement of the errors in the gyro sensor even though the gyro sensor data is highly covered. Figure 6 represents a different description of the HDR filter [29,30]. In the HDR filter, the term of $\omega_{\text {true }}$ is the ideal gyroscope data that does not include biases. However, the gyroscope includes $\varepsilon_{0}$ and $\varepsilon_{d}$ as it detects its data.

$$
\begin{aligned}
I_{i} & =I_{i-1}-\operatorname{SIGN}\left(\omega_{i-1}\right) i_{c} \\
\operatorname{SIGN}(x) & = \begin{cases}1 & \text { for } x>0 \\
0 & \text { for } x=0 \\
-1 & \text { for } x<0\end{cases}
\end{aligned}
$$

The term of $\varepsilon_{0}$ is the early bias value calculated by using the average of specific periods after removing motions in the gyroscope and the term of $\varepsilon_{d}$ is the bias value that is finely changed according to passage of time.

Although the Binary I-Controller is to be sensitively responded to a small error $(E)$, it considers the symbol of errors only because it should be insensitively responded to a large error (motions of the gyroscope). Thus, the Binary I-Controller can be expressed as Eq. (3). In Eq. (3), $i_{c}$ is a constant and the SIGN() function is presented as Eq. (4) in which the term of $\varepsilon_{0}$ can be calculated by the average of the accumulated data at a condition of fixing the gyroscope. Also, the finely changed bias $\left(\varepsilon_{d}\right)$ can be removed by the difference from the previous data $(t=n-1)$.

In the gyroscope data, the changes in remained biases in the bias, which is reduced by the HDR filter, were stabled using a low-frequency pass filter. 
S614 S.-H. Eom and E.-H. Lee / A study on the operation of rehabilitation interfaces in active rehabilitation exercises

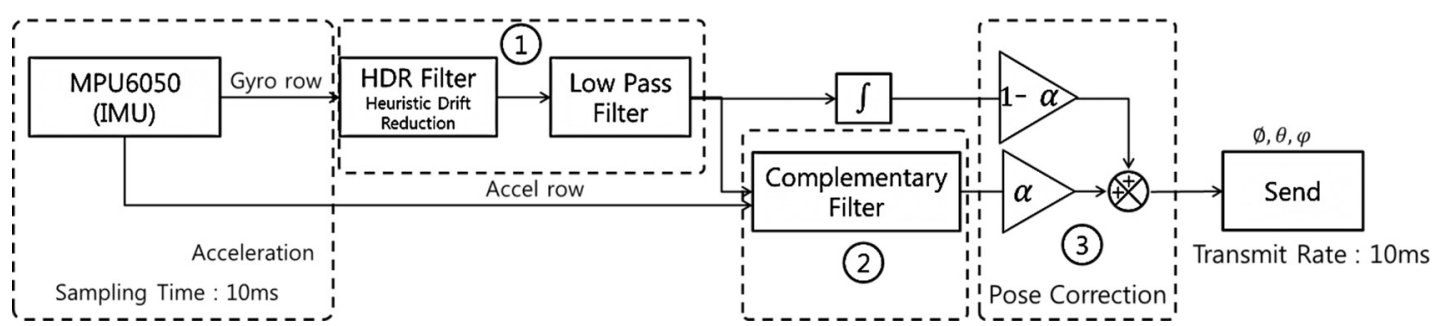

Fig. 7. Movement information module internal block diagram.

\subsection{Interface module for the lateral and bilateral rehabilitation exercise}

The interface module for the lateral and bilateral rehabilitation exercise was fabricated after deducing requirements of the exercise based on the previously proposed rehabilitation process. Three requirements were deduced as follows. First, it is possible to estimate the motion information in the unaffected side. Second, the estimation is to be performed with an error of $\pm 1^{\circ}$. Third, the measurement module is designed as a low-cost and small-size system. For satisfying these requirements, in this study a six-axis gyro + acceleration sensor was used to estimate the motion information in the unaffected and two different algorithms were merged to implement an algorithm that has low errors at a low-cost microprocessor. The merged algorithm represents a complementary filter that compensates angle estimation errors in the gyro and acceleration sensors and a heuristic drift reduction (HDR) filter for improving the cumulative error in the gyro sensor.

The structure of the proposed merged algorithm is presented in Fig. 7. The HDR filter was applied to minimize a bias value, which becomes a cause of integration errors, using (1). The complementary filter, which cancels both the physical noise in the acceleration sensor and the integration error of the gyroscope, was performed using (2). The weight value, which is presented at the integration of the output data of the complementary and HDR filters, was applied according to motional conditions of trainees using (3).

\subsection{Estimation of the shoulder and elbow joint angles for detecting upper limb positions}

Control variables in the upper limb rehabilitation robot using a bilateral interface are represented by joint state values of the shoulder and elbow joints in the unaffected part. The state of joints means the angle (degree of rotation) of the rotation axis in a joint. Although the estimation of the degree of rotation is precisely performed at the center of the rotation axis, in this study it is implemented under the following assumption because the estimation is not implemented by a specific subject but is an estimation of rehabilitation motions.

\subsubsection{State estimation of the upper limb shoulder joint (shoulder joint) in the unaffected part}

The secondary complementary filter and the motion information module with a HDR filter were used to detect the upper limb positions of the unaffected part in upper limb hemiplegic patients.

Although the installation of the motion information module at the rotation axis of a joint allows an easy acquisition of data, it shows a difficulty in installing it to the rotation axis precisely. Thus, in this study the positions of the brachium and forearm flap were configured in order to easily attach, detach, and move it by trainees. Figure 8 represents the installation point of the inertia measurement device. 


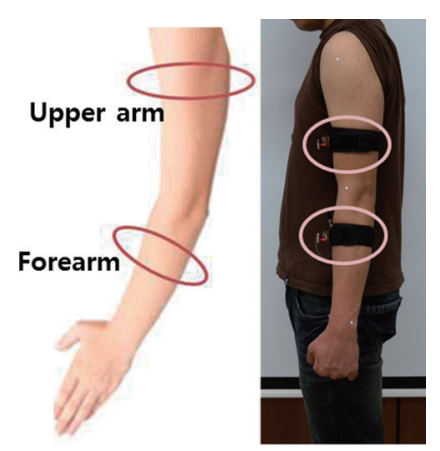

Fig. 8. Ideal attachment position of inertial measurement unit and real attachment position.

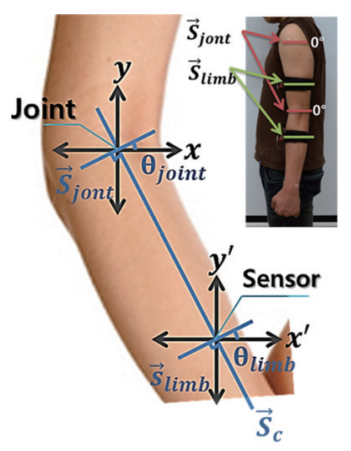

Fig. 9. The initial position (right) and inertial measurement unit detection method (left).

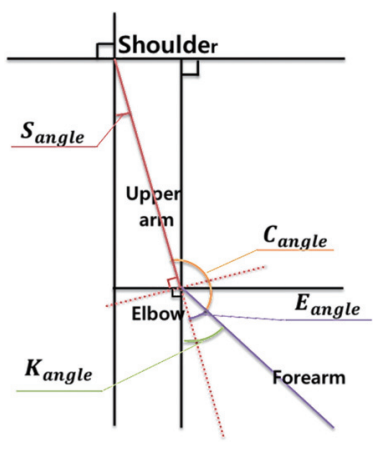

Fig. 10. Joint Estimation method.

As shown in Fig. 8, as the positions are detected from the brachium and forearm flap instead of the rotation axis of the joint, the detection is performed at an arc of the rotation motion. The detection methods in the brachium and forearm flap are presented in Fig. 9.

As presented in the left side in Fig. 9, the upper limb position angle detected from the initial position in which the shoulder and elbow are spreaded to a gravitation direction was assumed as $0^{\circ}$. Thus, the rotation axis $\left(\overline{S_{\text {joint }}^{\prime}}\right)$ of the joint is parallel to the linear element of the measurement axis $\left(\overline{S_{\text {linb }}^{\prime}}\right)$ of the inertia measurement device while both the joint rotation angle and the angle measured at the inertia measurement device in the initial position are determined as $0^{\circ}$ based on this assumption. Therefore, it can be assumed that the rotation angles of $\left(\theta_{\text {joint }}\right)$ and $\left(\theta_{\text {linb }}\right)$ measured at both the forearm flap and the brachium respectively are the same each other as corresponding angles. Based on this assumption, it is possible to estimate the Flexion/Extension, Abduction/Adduction, and Internal/External Rotation motions in the shoulder joint from the motion information module attached on the brachium.

\subsubsection{State estimation of the upper limb elbow joint in the unaffected part}

Based on the method proposed in Section 4.4.1, it was verified that the angles measured at both the center of a joint and the arc of its rotation are the same each other determined by using the initial position. As this method determines the rotation angle of the elbow joint based on the brachium, it is not possible to accurately measure the rotation angle of the elbow joint using a single motion information module even though the rotation angles of the shoulder joint are perfectly agree with each other. For correcting it, a method that estimates accurate elbow joint angles through complementing it with the shoulder joint angle estimation angle is used as shown in Fig. 10.

The upper limb position angle detected from the motion information module of the brachium is $S_{\text {angle }}$ and is the same as the rotation angle of the shoulder joint. However, as the upper limb position angle detected from the motion information module of the forearm flap, $K_{\text {angle }}$ includes the rotation angle $\left(S_{\text {angle }}\right)$ of the shoulder joint, the estimation of the elbow joint rotation angle $\left(E_{\text {angle }}\right)$ is performed by removing the shoulder joint rotation angle. The estimation of the elbow joint rotation angle is presented as Eq. (5).

$$
E_{\text {angle }}=K_{\text {angle }}-S_{\text {angle }}
$$


Table 2

The specifications of movement information module

\begin{tabular}{ll}
\hline & Specification \\
\hline MCU & STM32F405RGT \\
IMU & MPU6050 (6DoF) \\
Communication & bluetooth $(2.4 \mathrm{GHz})$ \\
Battery & 3.7 V LiPo 130 mAh \\
\hline
\end{tabular}

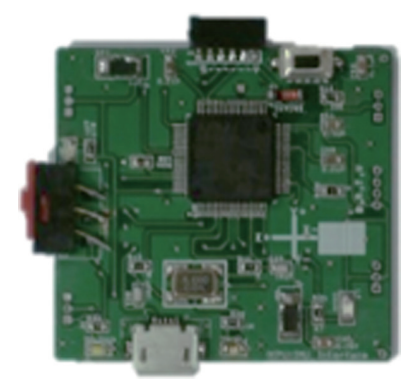

Fig. 11. Movement information module.

\section{Results and discussion}

In this study an interface method that allows bilateral exercises is proposed to implement active rehabilitation exercises in upper limb hemiplegic patients.

For verifying the proposed method, the reliability of the motion information module was verified through an experiment that examines the performance of both the HDR and the complementary filters. Then, an encoder based device that is similar to the upper limb of human beings was fabricated to verify the joint angle estimation method based on the correlation analysis between the shoulder joint and the elbow joint. Figure 11 shows the motion information module used in the verification experiment and Table 2 represents its specification.

\subsection{Experiment and results of the motion information module}

The experimental environment and method of verifying the performance of the HDR filter are presented in Fig. 12. The experimental environment, as shown in Fig. 12, was configured and the pendulum motions were generated in order to compare and analyze the degree of generating integration errors before and after applying the HDR filter. Figure 13 represents the results of this experiment.

The data before applying the HDR filter, as presented in Fig. 13, showed divergences in data compared to the reference value after a given period due to integration errors and the errors were largely reduced as a agreement ratio of $96.52 \%$ after applying the HDR filter compared to that of the encoder. The sensor drift after applying the HDR filter was about $0.034^{\circ}$ per hour and was a reasonable error for estimating motion information as the rehabilitation exercise duration is about 30 minutes.

The experimental environment of verifying the complementary filter was the same as the experiment of the HDR filter. In the verification, the performance of the filter was examined by comparing the angles between the complementary filter and the encoder.

As shown in Fig. 14 the average error angle between the complementary filter and the encoder was $0.041^{\circ}$ in the results of the complementary filter.

In addition, it was verified that the error correction between the HDR and the complementary filters was performed based on these two experiments. Thus, the performance of the motion information module was also verified.

\subsection{Experiment of the correlation analysis between the shoulder and the elbow joints}

The proposed method is to be verified by determining the accuracy of the joint angle estimation method based on the correlation analysis between the shoulder and the elbow joints. The experimental environment was configured by designing an encoder based device that is similar to the upper limb of human beings as shown in Fig. 15. 


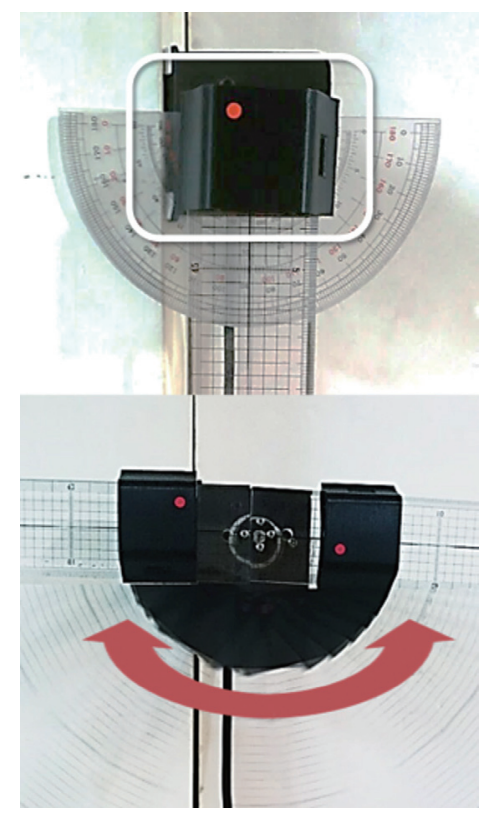

Fig. 12. HDR filter performance verification test results.

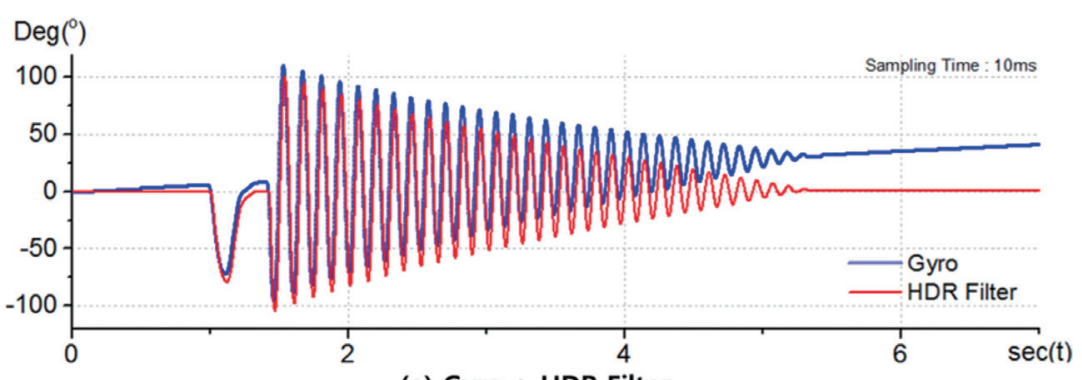

(a) Gyro + HDR Filter

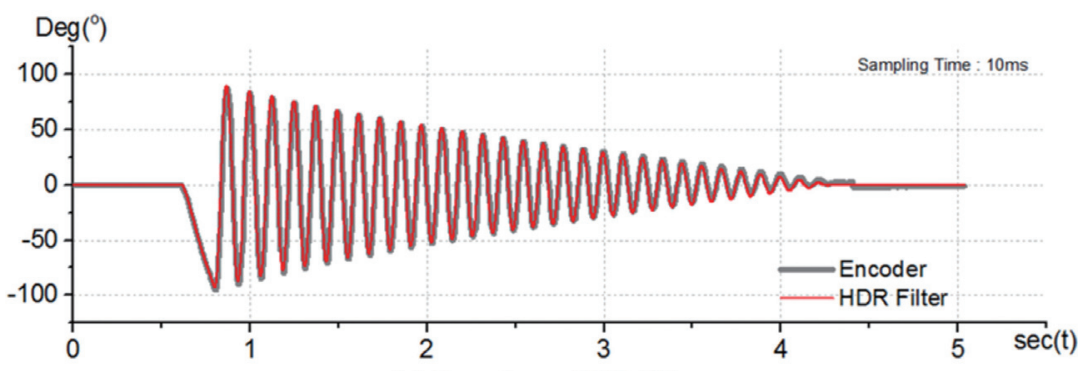

(b) Encoder + HDR Filter

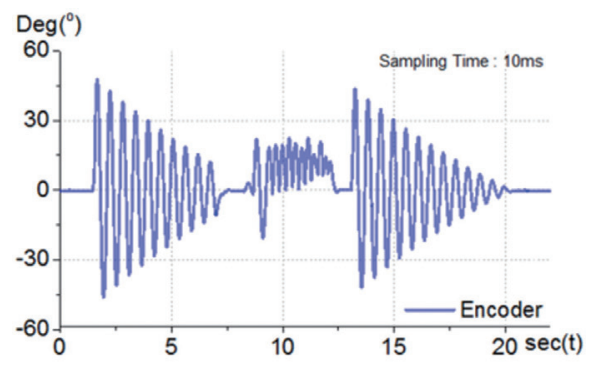

(a) Encoder

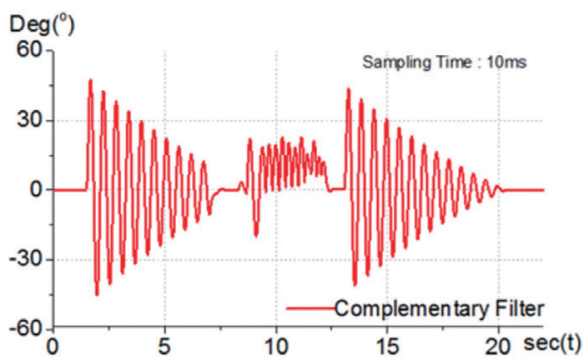

(b) Complementary Filter

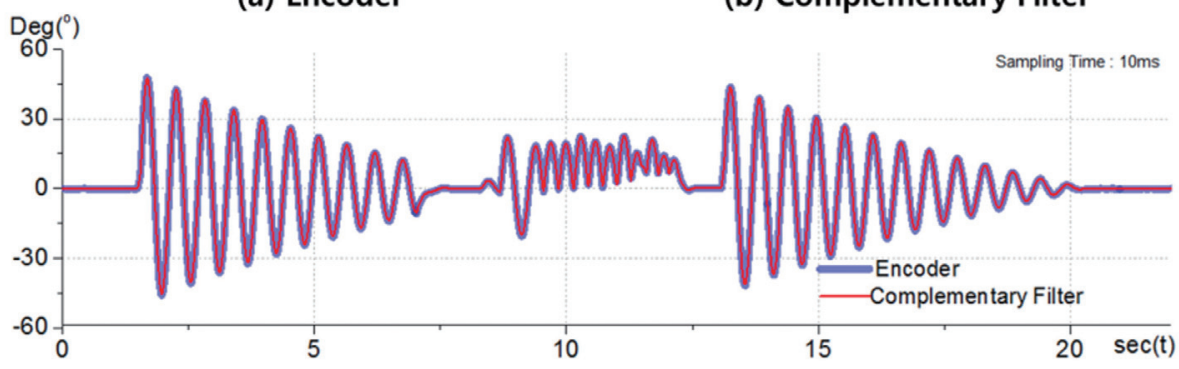

(c) Encoder + Complementary Filter

Fig. 14. Complementary filter performance verification test results.

Encoder angles were used as the data for the practically moved shoulder and elbow joints. Then, the verification was implemented by the comparison between the joint angles estimated in the motion information module and the practical joint rotation angles. In the results of the experiment shown in Fig. 16, the angles detected from the motion information module of the brachium and the estimation 

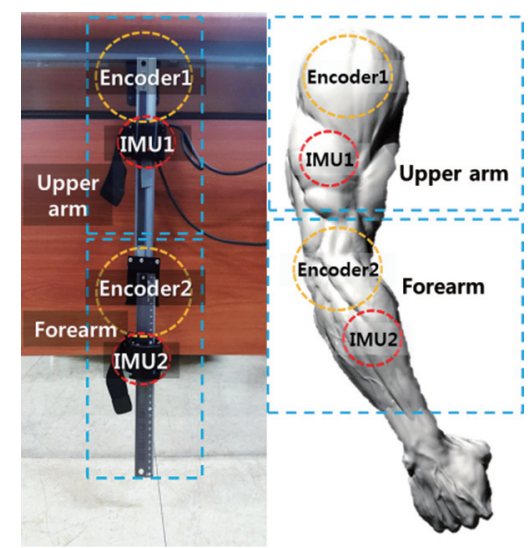

Fig. 15. A similar mechanism design with the human upper extremity.
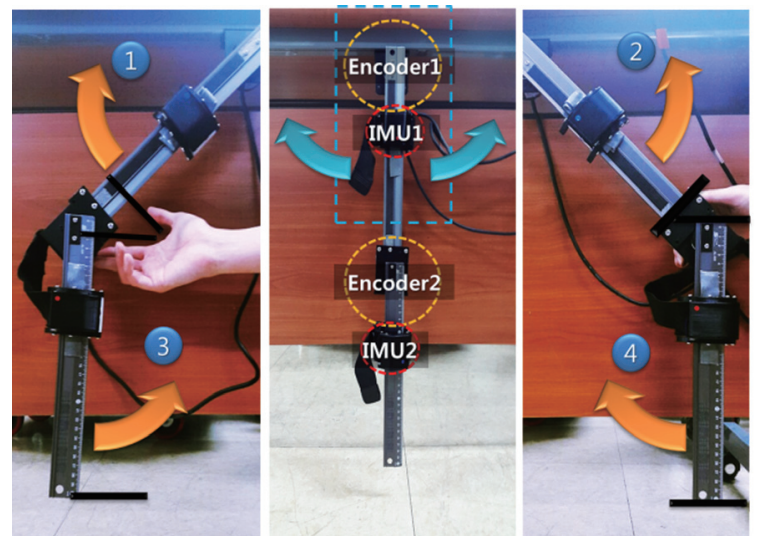

Fig. 16. Single shoulder movement test.

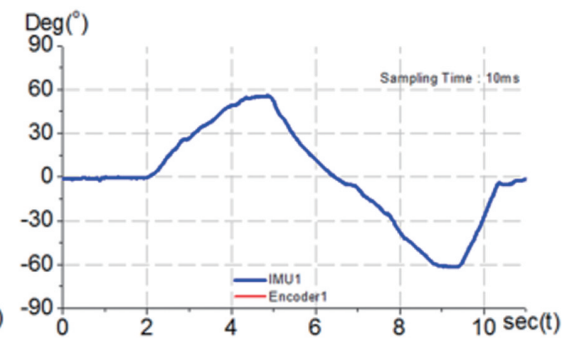

(b) Shoulder(IMU1)

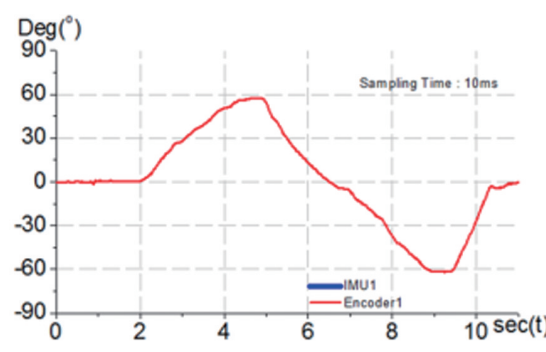

(a) Shoulder(Encoder1)

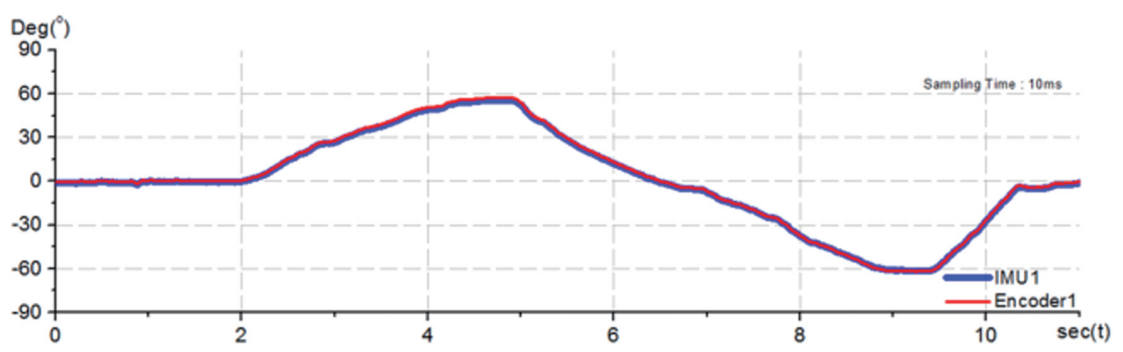

(c) Shoulder(IMU1 + Encoder1)

Fig. 17. Comparing the shoulder movement estimation values.

graph of the shoulder joint angles, which are induced from Encoder 1, are presented in Fig. 17.

As represented in Fig. 17, it was verified that the shoulder joint angles were homogeneously measured due to the agreement between the reference point of Encoder 1 and the gravity reference of the motion information module (IMU1).

In Fig. 18, however, there are no changes in the rotation angles in the motion information module even though the elbow joint rotation angles are varied because the reference point of Encoder 2 is not the same as the gravity reference of the motion information module (IMU2).

In Figs 17 and 18, the angles measured at the upper limb motion information modules (IMU1) and the forearm flap motion information module (IMU2) are and respectively. Thus, the results of applying the joint angle estimation method using Eq. (5) are presented in Fig. 19. 


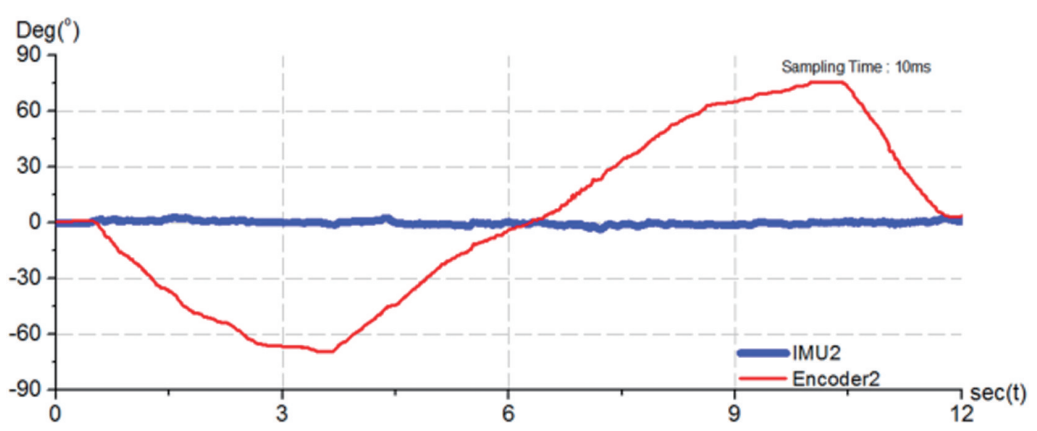

Fig. 18. Comparing the elbow movement estimation values.

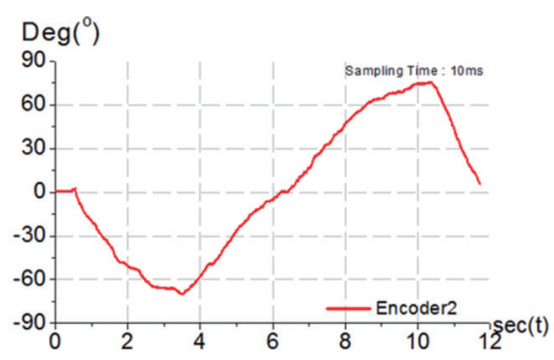

(a) Elbow(Encoder2)

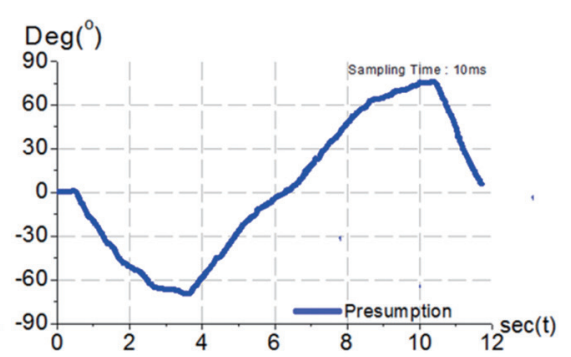

(b) Shoulder(Presumption)

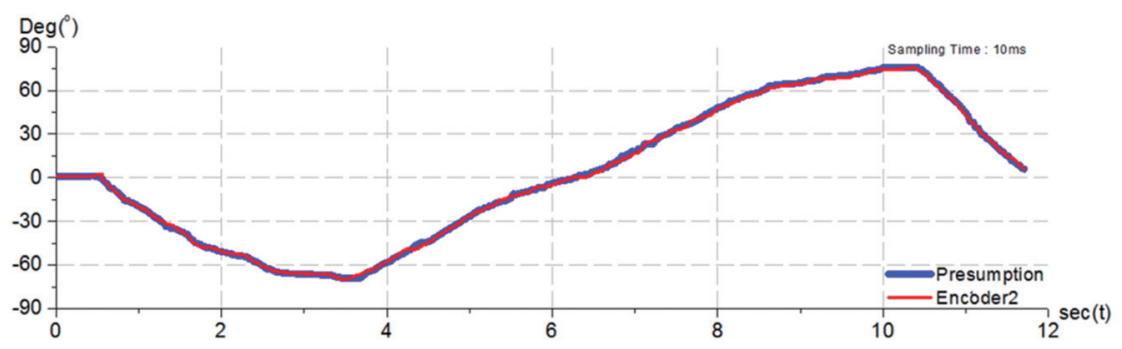

(c) Shoulder(Presumption + Encoder2)

Fig. 19. Comparing estimation values after applying the joint estimation method.

It was verified that the angle measured in the motion information module of the forearm flap includes the shoulder joint rotation angle based on the analysis of the correlation between the shoulder and the elbow joints. Therefore, the average error angle determined by the comparison between the angles obtained by using Eq. (4) and the practical elbow joint angles was $0.299^{\circ}$. Thus, it was verified that the elbow joint angle can be estimated using the joint angle estimation method.

The shoulder and elbow joint angles estimated by using the proposed method can be used as control variables in a upper limb rehabilitation robot. Because the robot based on these variables is able to express the motion of the unaffected part in upper limb hemiplegic patients to the affected part, it can implement bilateral exercises.

\subsection{Verification of the rehabilitation scenario}

As shown in Figs 20-22, an exoskeleton robot was used to verify the rehabilitation scenario proposed in this study. 

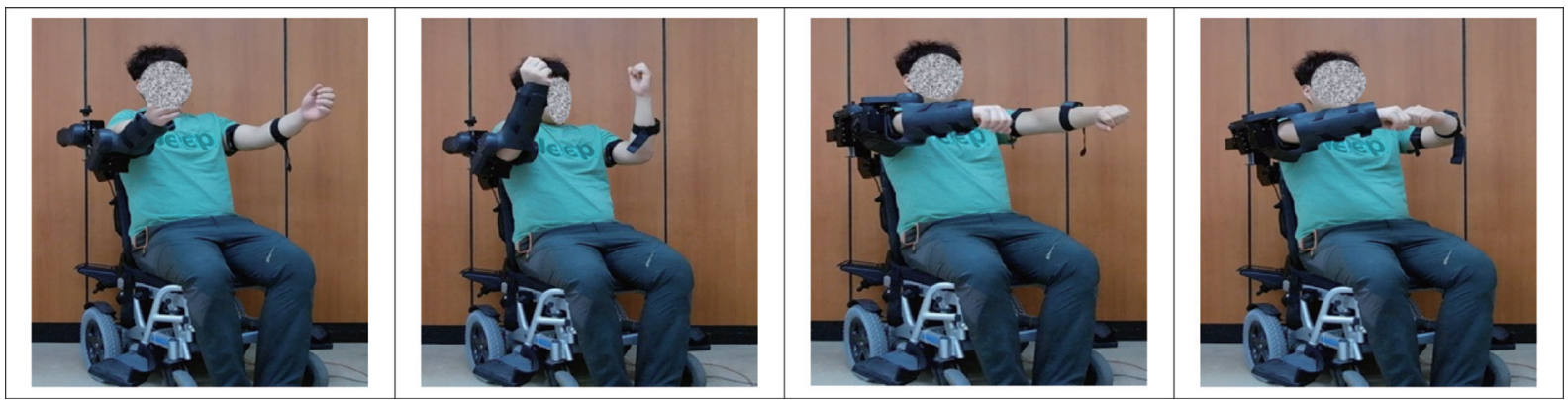

Fig. 20. The motions of first step of the proposed scenario.
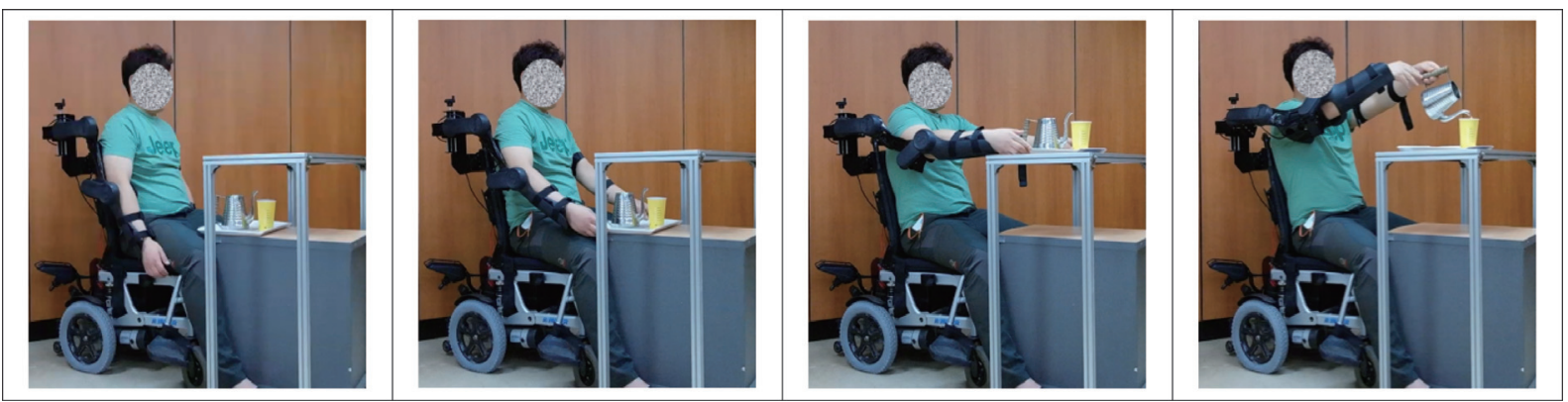

Fig. 21. The motions of second step of the proposed scenario.
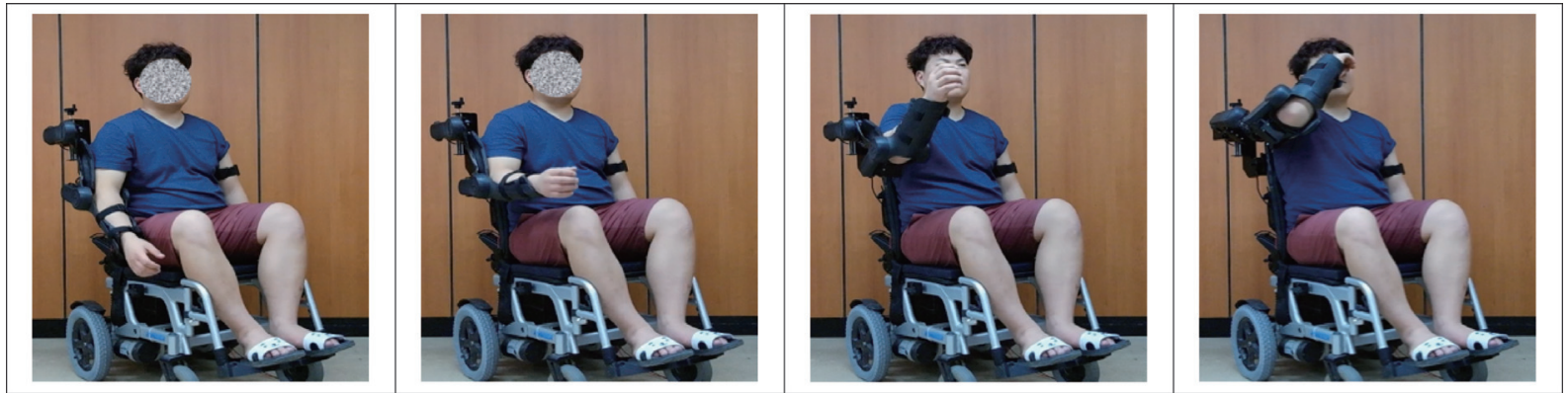

Fig. 22. The motions of third step of the proposed scenario.

Figure 20 represents the first step of the proposed scenario in which the affected part expresses the motion of the unaffected part. It is similar to the lateral rehabilitation exercise. The difference between this scenario and the conventional CPM device is autonomous exercises based on the willingness of patients instead of applying formal exercises.

Figure 21 shows the second step of the scenario that includes various muscle and nerve aware exercises through bilateral exercises. The reason that a single rehabilitation robot can implement both lateral and bilateral exercises is based on the expression method proposed in this study in which the affected part expresses the motion of the unaffected part.

Figure 22 shows combination motions of one and both hands usually happened in daily life. The reason that requires this exercise is to prepare different situations in daily life because there are lots of unexpected situations in our daily life. Thus, it is an exercise for adopting different environments. 


\section{Conclusion}

In this study an interface method and a rehabilitation scenario that implement lateral and bilateral exercises in a upper limb rehabilitation robot were proposed to support active rehabilitation exercises of upper limb hemiplegic patients. The proposed method is an interface method that expresses the estimated position of the unaffected part of patients to a rehabilitation exercise robot attached to the affected part and is different from the conventional robot and device based exercise methods. Also, it allows autonomous exercises of patients. For achieving it, an inertia measurement device was developed and used. Also, the accuracy of the estimation was ensured through applying complementary and HDR filters in order to easily estimate upper limb motions. In addition, a method that uses individual modules attached at both the brachium and the forearm flap in the unaffected part for applying it to an elbow joint angle detection method and to control variables of a upper limb rehabilitation robot was proposed.

For verifying the proposed method, experiments that verify the motion information module and analyze the correlation between the shoulder and the elbow joints were configured.

The experiments to examine the motion information module were configured by the verifications of HDR and complementary filters and the results were determined by reductions in the integration errors of the gyroscope based on the comparison between before and after applying the HDR filter. The experiment of the complementary filter was implemented by comparing the results with the angles detected from the encoder and the average error angle was confirmed by $0.041^{\circ}$.

In the experiment of analyzing the correlation between the shoulder and the elbow joints, an encoder based device that is similar to the upper limb of human beings was designed in which the angles measured at the encoder were assumed as practical joint rotation angles in this experiment. In the results, in the case of the angles measured by using the motion information module (IMU1) in the brachium, the rotation angles of the shoulder joint showed the same values because the reference point of the encoder was the same as both the reference axis and the gravity reference axis. In the case of the motion information module (IMU2) in the forearm flap, however, the rotation angles were different from the practical elbow joint rotation angles because the reference axis of IMU2 was not agreed with the reference point of the encoder. It is due to the fact that the rotation angles of the shoulder joint are simultaneously detected. In the results of applying the proposed elbow joint estimation method, the average error angle was determined by $0.299^{\circ}$ compared to that of the practical elbow joint rotation angle.

In addition, motions of rehabilitation exercises for each rehabilitation process were implemented to verify the rehabilitation scenario proposed in this study. Also, a camera analysis method was used to verify such motions and the results were compared and verified according to the scenario. In the results of the comparison, the scenario was well accomplished.

It is possible to apply the method proposed in this study to the control variables in different upper limb rehabilitation exercise robots. Thus, it is expected that patients centered active lateral/bilateral rehabilitation exercises can be performed through this interface method.

The proposals in this study include the estimation of the partly limited elbow and shoulder joint angles and require additional researches including the control of robot joint angles and the agreement between the estimated and practical robot operation angles in order to perform patients centered active rehabilitation exercises.

\section{Acknowledgments}

This research was supported by the MSIP(Ministry of Science, ICT and Future Planning), Korea, under the C-ITRC (Convergence Information Technology Research Center) (IITP-2015-H8601-15-1003) supervised by the IITP (Institute for Information \& Communications Technology Promotion). 


\section{References}

[1] D. Erol, Coordinated control of assistive robotic devices for activities of daily living tasks, IEEE Transactions on Neural Systems and Rehabilitation Engineering, Vol. 16, No. 3, June 2008, pp. 278-285.

[2] S. Zhang, Probabilistic learning from incomplete data for recognition of activities of daily living in smart homes, IEEE Transactions On Information Technology in Biomedicine, Vol. 16, No. 3, May 2012, pp. 454-462.

[3] R. Colombo, F. Pisano, S. Micera, A. Mazzone, C. Delconte, M.C. Carrozza, P. Dario and G. Minuco, Robotic techniques for upper limb evaluation and rehabilitation of stroke patients, IEEE Transactions On Neural Systems and Rehabilitation Engineering, Vol. 13, No. 3, September 2005, pp. 311-323.

[4] P.R. Culmer, A.E. Jackson, S. Makower, R. Richardson, J.A. Cozens, M.C. Levesley and B.B. Bhakta, A control strategy for upper limb robotic rehabilitation with a dual robot system, IEEE/ASME Transactions on Mechatronics, Vol. 15, No. 4, August 2010, pp. 575-585.

[5] R.J. Sanchez, J. Liu, S. Rao, P. Shah, R. Smith, T. Rahman, S.C. Cramer, J.E. Bobrow and David J. Reinkensmeyer, Automating arm movement training following severe stroke: functional exercises with quantitative, IEEE Transactions on Neural Systems and Rehabilitation Engineering, Vol. 14, No. 3, September 2006, pp. 378-388.

[6] H.I. Krebs, S. Member, B.T. Volpe, D. Williams, J. Celestino, S.K. Charles, D. Lynch and N. Hogan, Robot-aided neurorehabilitation: a robot for wrist rehabilitation, IEEE Transactions on Neural Systems and Rehabilitation Engineering, Vol. 15, No. 3, September 2007, pp. 327-335.

[7] R. Colombo, I. Sterpi, A. Mazzone, C. Delconte, G. Minuco and F. Pisano, Measuring changes of movement dynamics during robot-aided neurorehabilitation of stroke patients, IEEE Transactions on Neural Systems and Rehabilitation Engineering, Vol. 18, No. 1, February 2010, pp. 75-85.

[8] A. Duschau-Wicke, J. von Zitzewitz, A. Caprez, L. Lünenburger and R. Riener, Path control: a method for patientcooperative robot-aided gait rehabilitation, IEEE Transactions on Neural Systems and Rehabilitation Engineering, Vol. 18, No. 1, February 2010, pp. 38-48.

[9] K.-J. Kim, M.-S. Kang, Y.-S. Choi, J. Han and C. Han, Conceptualization of an exoskeleton ContinuousPassive Motion (CPM) device using a link structure, 2011 IEEE International Conference on Rehabilitation Robotics Rehab Week Zurich, ETH Zurich Science City, Switzerland, June 29-July 1, 2011.

[10] H.I. Krebs, N. Hogan, M.L. Aisen and B.T. Volpe, Robot-aided neurorehabilitation, IEEE Transactions on Rehabilitation Engineering, Vol. 6, No. 1, March 1998, pp. 75-87.

[11] S.M. Waller and J. Whitall, Fine motor control in adults with and without chronic hemiparesis: baseline comparison to nondisabled adults, and effects, of bilateral arm training, Arch Phys Med Rehabil Vol. 85, July 2004, pp. 1076-1083.

[12] J.-J. Chang, W.-L. Tung, MS, W.-L. Wu, M.-H. Huang and F.-C. Su, Effects of robot-aided bilateral force-induced isokinetic arm training combined with conventional rehabilitation on arm motor function in patients with chronic stroke, Arch Phys Med Rehabil Vol. 88, October 2007, pp. 1332-1337.

[13] O. Schuhfried, C. Kollmann and T. Paternostro-Sluga, Excitability of chronic hemiparetic muscles: determination of chronaxie values and strength-duration curves and its implication in functional electrical stimulation, IEEE Transactions on Neural Systems and Rehabilitation Engineering, Vol. 13, No. 1, March 2005, pp. 105-109.

[14] J. Solana, C. Cáceres, A.G. a-Molina, E. Opisso, T. Roig, J.M. Tormos and E.J. Gómez, Improving brain injury cognitive rehabilitation by personalized telerehabilitation services: guttmann neuropersonal trainer, IEEE Journal Of Biomedical and Health Informatics, Vol. 19, No. 1, January 2015, pp. 124-131.

[15] L. Yu, L. Guo, X. Gu, J. Fu and Q. Fang, LVQ neural network applied for upper limb motion recognition, Bioelectronics and Bioinformatics (ISBB), 2011 International Symposium on, 2011, pp. 151-154.

[16] G. Kwakkel, B.J. Kollen and A.J.H. Prevo, Probability of regaining dexterity in the flaccid upper limb impact of severity of paresis and time since onset in acute stroke, Epub, 7 Aug 2003, pp. 2181-2186.

[17] M.J. Johnson, H.F. Machiel Van der Loos, C.G. Burgar, P. Shor and L.J. Leifer, Experimental results using force-feedback cueing in robot-assisted stroke therapy, IEEE Transactions on Neural Systems and Rehabilitation Engineering, Vol. 13, No. 3, September 2005, pp. 335-348.

[18] C.E. Lang, J.M. Wagner, D.F. Edwards and A.W. Dromerick, Upper extremity use in people with hemiparesis in the first few weeks after stroke, JNPT, Vol. 31, June 2007, pp. 56-63.

[19] A.R. Luft, S. McCombe-Waller, J. Whitall, L.W. Forrester, R. Macko, J.D. Sorkin, J.B. Schulz, A.P. Goldberg and D.F. Hanley, Repetitive bilateral arm training and motor cortex activation in chronic stroke, JAMA, Vol. 292, No. 15, October20, 2004, pp. 1853-1861.

[20] G.N. Lewis and E.J. Perreault, An assessment of robot-assisted bimanual movements on upper limb motor coordination following stroke, IEEE Transactions on Neural Systems and Rehabilitation Engineering, Vol. 17, No. 6, December 2009, pp. 595-604.

[21] P.S. Lum, C.G. Burgar and P.C. Shor, Evidence for improved muscle activation patterns after retraining of reaching movements with the mime robotic system in subjects with post-stroke hemiparesis, IEEE Transactions on Neural Systems and Rehabilitation Engineering, Vol. 12, No. 2, June 2004, pp. 186-194. 
[22] S.V. Adamovich, G.G. Fluet, A.S. Merians, A. Mathai and Q. Qiu, Incorporating haptic effects into threedimensionalvirtual environments to train the hemiparetic upper extremity, IEEE Transactions on Neural Systems and Rehabilitation Engineering, Vol. 17, No. 5, October 2009, pp. 512-520.

[23] J.F. Vasconcelos, B. Cardeira, C. Silvestre, P. Oliveira and P. Batista, Discrete-time complementary filters for attitude and position estimation: design, Analysis and Experimental Validation, IEEE Transactions on Control Systems Technology, Vol. 19, No. 1, January 2011, pp. 181-198.

[24] D. Firoozi and M. Namvar, Analysis of gyro noise in non-linear attitude estimation using a single vector measurement, IET Control Theory Appl, Vol. 6, No. 14, 2012, pp. 2226-2234.

[25] M.-D. Hua, G. Ducard, T. Hamel, R. Mahony and K. Rudin, Implementation of a nonlinear attitude estimator for aerial robotic vehicles, IEEE Transactions on Control Systems Technology, Vol. 22, No. 1, January 2014, pp. 201-213.

[26] D. Kim and R.T. M'loskey, Spectral analysis of vibratory gyro noise, IEEE Sensors Journal, Vol. 13, No. 11, November 2013, pp. 4361-4374.

[27] J.F. Vasconcelos, C. Silvestre, P. Oliveira, P. Batista and B. Cardeira, Discrete time-varying attitude complementary filter, 2009 American Control Conference Hyatt Regency Riverfront, St. Louis, MO, USA, June 10-12, 2009, pp. 4056-4061.

[28] R. Mahony, S. Member, T. Hamel and J.-M. Pflimlin, Nonlinear complementary filters on the special orthogonal group, IEEE Transactions on Automatic Control, IEEE, Vol. 53, No. 5, June 2008, pp. 1203-1218.

[29] Q. Zhang, L. Wan and Y. Pang, Fault-tolerant GPS/DR integrated navigation system based on heuristic reduction of MEMS inertial measurement unit drift, Information Engineering and Computer Science, 2009. ICIECS 2009. International Conference, pp. 1-4.

[30] H. Qian, Q. Xia, B. Liu, D. An and X. Peng, Fuzzy heuristic reduction of gyro drift in gyrobased mobile robot tracking, Information Engineering and Computer Science, 2009. ICIECS 2009. International Conference, pp. 1-4. 